\title{
TRATAMENTO CONSERVADOR EM CERATOCÍSTICO ODONTOGÊNICO UTILIZANDO UM DISPOSITIVO INTRAÓSSEO: RELATO DE CASO
} Conservative treatment of odontogenic keratocystic using an intraosseous device: case report

\author{
Luiz Carlos Moreira Júnior ${ }^{\mathrm{a}}$ \\ Luis Felipe Barbosa da Silva ${ }^{b}$ \\ Júlio Holanda Alves de Souzac \\ Gabriel Gomes da Silva ${ }^{d}$ \\ Juliana Campos Pinheiro ${ }^{\mathrm{e}}$ \\ Rafaella Bastos Leite ${ }^{f}$
}

\begin{abstract}
RESUMO
O ceratocisto odontogênico (CO) classificado pela Organização Mundial da Saúde (OMS) como uma lesão cística de desenvolvimento benigna, se destaca entre as demais lesões odontogênicas em virtude do comportamento biológico potencialmente agressivo, com tendência a recorrências. O presente trabalho tem como objetivo, relatar um caso CO em mandíbula, bem como discutir aspectos relacionados aos achados radiográficos e tipos de tratamentos dessa lesão. Paciente do sexo masculino, leucoderma, exibia uma lesão radiolúcida circunscrita, e consistência endurecida, com duração de seis meses, medindo aproximadamente $1 \mathrm{~cm}$ de extensão em região posterior de mandíbula esquerda. Sob a hipótese clínica de cisto odontogênico de desenvolvimento, foi realizada
\end{abstract}

biópsia incisional. Com base no diagnóstico definitivo de CO após realização do exame histopalógico, foi realizada a remoção das unidades dentárias acometidas e colocado um dispositivo intraósseo para que houvesse a descompressão do CO. Após seis meses foi observada a regressão parcial da lesão, e o dispositivo ósseo foi retirado e o remanescente da lesão removida realizando osteotomia periférica. Após um ano de proservação não foi observada recidiva do CO até o presente momento. Os achados do presente caso ressaltam a importância do diagnóstico clínico e radiográfico, assim como o correto tratamento cirúrgico, enaltecendo a importância de um exame clínico minucioso da cavidade oral.

Palavras-chave: Cistos ósseos. Cirurgia bucal. Patologia.

\footnotetext{
${ }^{a}$ Cirurgião Bucomaxilofacial, Graduado pela Universidade Federal do Rio Grande do Norte, Natal, RN, Brasil.

b Graduando em Odontologia, Universidade Federal do Rio Grande do Norte, Natal, RN, Brasil.

${ }^{c}$ Graduando em Odontologia, Universidade Federal do Rio Grande do Norte, Natal, RN, Brasil.

d Graduando em Odontologia, Universidade Federal do Rio Grande do Norte, Natal, RN, Brasil.

${ }^{\text {e }}$ Cirurgiã-Dentista. Mestra em Patologia Oral, Universidade Federal do Rio Grande do Norte, Natal, RN, Brasil.

${ }^{\mathrm{f}}$ Cirurgiã-Dentista. Doutora em Patologia Oral, Universidade Federal do Rio Grande do Norte, Natal, RN, Brasil.
}

Autora de correspondência: Juliana Campos Pinheiro - E-mail: juliana.patologia92@gmail.com

Data de envio: 29/08/2019 | Data de aceite: 13/09/2019 


\begin{abstract}
The odontogenic keratocyst (OK) classified by the World Health Organization (WHO) as a benign developmental cyst, stands out among other odontogenic lesions because of its potentially aggressive biological behavior, prone to recurrence. This work aims to report a case of OK in the mandible, as well as discuss aspects related to the radiographic findings and ways of treatment. Male patient, white skinned, had shown a circumscribed, radiolucid lesion with a hardened consistency, six months old, measuring approximately 1 centimeter wide on the posterior left mandible region. Under the clinical hypothesis of odontogenic developmental cyst, an incisional biopsy was successfully made. Based on the definitive diagnosis of OK after the histopathological exam, the removal of the affected teeth was then realized, as well as the implementation of an intraosseous device so the decompression of the OK could happen. After six months a partial regression of the lesion was observed, the intraosseous device was then removed and the remaining lesion was removed through a peripherical osteotomy. After a year of proservation, no recurrence of the OK was observed to this moment. The present case findings stress the importance of a clinical and radiographic diagnosis, as well as the right surgical treatment, praising the importance of a thorough clinical exam of the oral cavity.
\end{abstract}

Keywords: Bone cysts. Surgery oral. Pathology.

\title{
INTRODUÇÃO
}

O ceratocisto odontogênico (CO) foi descrito pela primeira vez em 1956. Em 2005 a Organização Mundial de Saúde (OMS) classificou o CO como tumor odontogênico ceratocístico devido ao seu comportamento clínico agressivo e as suas altas taxas de recidiva local ${ }^{1}$. Em 2017 a OMS voltou a classificá-lo como um cisto odontogênico de desenvolvimento, visto que em alguns casos a lesão não exibia comportamento agressivo, além de histopatologicamente a lesão apresentar características císticas².

O CO é um uma lesão cística intraóssea de caráter benigno, contudo seu tratamento requer cuidados especiais devido aos seus aspectos histopatológicos específicos associados às taxas de recidiva. Esta lesão cística tem predileção pelo sexo masculino, acometendo indivíduos que estão entre a $2^{\mathrm{a}}$ e $3^{\mathrm{a}}$ décadas de vida. A mandíbula é de três a quatro vezes mais acometida do que a maxila, em região de terceiros molares ${ }^{3}$.

Radiograficamente o CO apresenta-se como uma lesão radiolúcida uni ou multilocular, bem circunscrita, podendo envolver dentes não erupcionados, exibindo reabsorção radicular e extrusão dos dentes envolvidos ${ }^{4}$.

A etiopatogênia para o seu desenvolvimento odontogênico pode estar associada aos remanescentes da lâmina dentária ou proliferação das células da camada basal do epitélio oral ${ }^{5}$.

Histopatologicamente a lesão é revestida por epitélio pavimentoso estratificado paraceratinizado corrugado e circundada por um tecido conjuntivo fibroso denso, onde pode ser observada a presença de cistos satélites, que podem estar associados as altas taxas de recidiva da lesão, além disso, a recidiva pode estar associada ao limite plano entre tecido epitelial lesional e conjuntivo da cápsula, tornando a lesão friável e de difícil curetagem ${ }^{6}$.

Devido à alta taxa de recidiva do CO, que varia de $25 \%$ à $60 \%$, o tratamento ainda é controverso, entretanto existem dois tipos: o tratamento conservador, caracterizado pela descompressão e a marsupialização seguida da enucleação com ou sem curetagem da lesão e os tratamentos mais agressivos onde é realizado ostectomia periférica, crioterapia e curetagem química com solução de Carnoy, entretanto, por mais que um tratamento agressivo seja mais seguro, evitando a recidiva do CO, podem ocorrer danificação de estruturas nobres do paciente, como vasos sanguíneos e nervos ${ }^{7}$. 
Quando o diagnóstico de CO é confirmado histopatologicamente, deve-se investigar a Síndrome de Gorlin-Goltz, pois além de OC, o paciente pode apresentar carcinoma basocelular, hipertelorismo ocular e costelas bífidas. O prognóstico do CO, ainda é controverso, devido ao seu caráter recidivante ${ }^{8}$.

O presente trabalho tem como objetivo relatar um caso CO em mandíbula, bem como discutir aspectos relacionados aos achados radiográficos e o tipo de tratamento estabelecido para esta lesão.

\section{RELATO DE CASO}

Paciente, sexo masculino, leucoderma, 40 anos, apresentava uma lesão intraóssea unilocular, circunscrita, assintomática, medindo aproximadamente $1 \mathrm{~cm}$ de diâmetro em região posterior de mandíbula esquerda, com tempo de evolução de seis meses detectada através de radiografia panorâmica (Figura 1A). No exame intraoral, não se observava alterações dignas de nota.

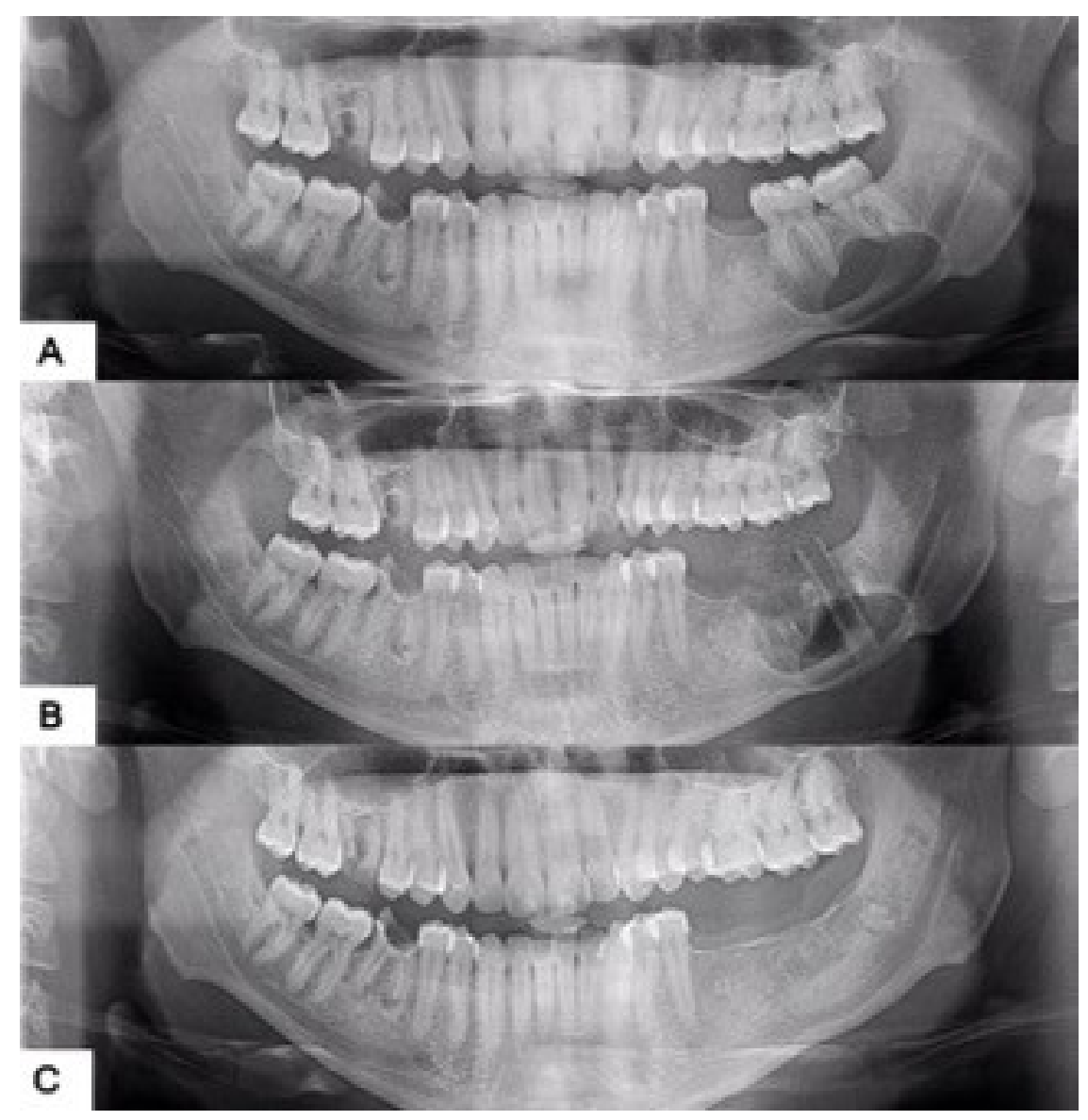

Figura 1: A) Lesão intraóssea, circunscrita, medindo aproximadamente 1,0cm de diâmetro em região posterior de mandíbula esquerda; B) Dispositivo intraósseo instalado na lesão; C) Proservação após 1 ano sem presença de recidiva local até o presente momento. 
Foi realizado o teste de vitalidade pulpar das unidades dentárias acometidas 37 e 38, e os mesmos responderam positivamente. Sob a hipótese diagnóstica de cisto de natureza odontogênica, foi realizada uma biópsia incisional e encaminhada para a análise histopatológica. Microscopicamente, observou-se presença de cavidade patológica revestida por epitélio pavimentoso estratificado paraceratinizado corrugado, apresentando espessura uniforme, variando entre seis e oito camadas de células. As células basais se apresentam colunares com núcleos dispostos em paliçada, exibindo hipercromatismo nuclear e circundada por uma espessa cápsula de tecido conjuntivo fibroso, apresentando um leve infiltrado inflamatório mononuclear (Figura 2).
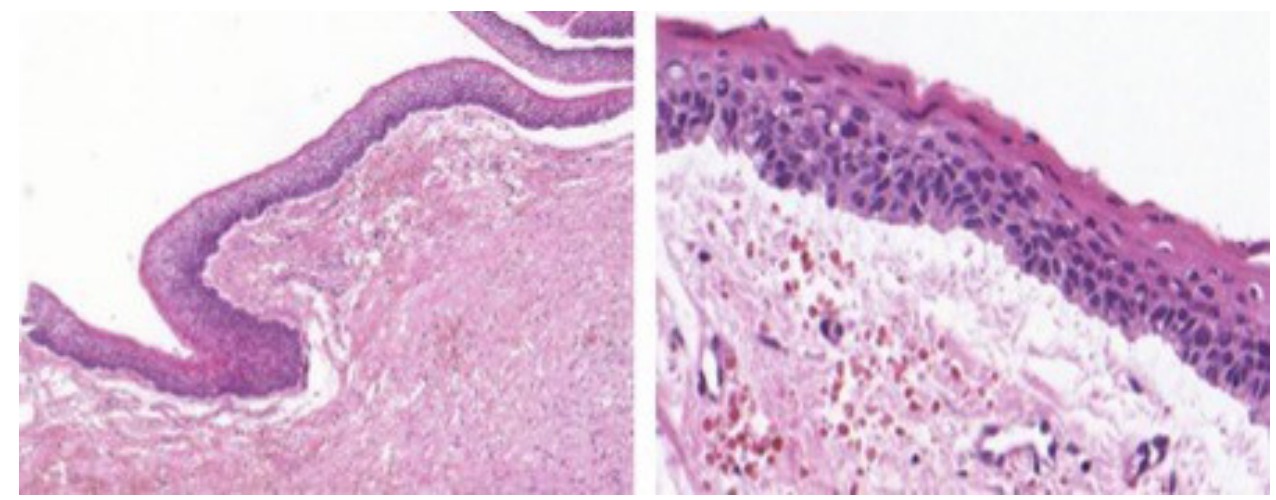

Figura 2: Cavidade patológica revestida por epitélio pavimentoso estratificado paraceratinizado corrugado, apresentando espessura uniforme, variando entre seis e oito camadas de células e camada basal apresentando hipercromatismo nuclear. A lesão era circundada por uma cápsula espessa de tecido conjuntivo fibroso, apresentando um leve infiltrado inflamatório mononuclear.

Ao se confirmar o diagnóstico histopatológico de CO, foi realizada a exodontia dos elementos dentários envolvidos, pela possibilidade de permanecer remanescentes epiteliais entre as raízes e recidiva da lesão e para se obter melhor acesso cirúrgico a lesão. Foi inserido um dispositivo intraósseo para descomprimir a da lesão cística (Figura 1B), o paciente não relatou desconforto com o uso do mesmo e não foram observadas complicações pós-operatória. O paciente foi orientado em relação aos cuidados de higiene oral, sendo instruído a manter a escovação normal durante três vezes ao dia, não havendo presenças de infecções, o paciente foi colaborativo. Após seis meses foi observada a regressão parcial da lesão, e o dispositivo ósseo foi retirado e o remanescente da lesão removida realizando osteotomia periférica. Após um ano de proservação não foi observada recidiva do CO até o presente momento (Figura 1C). O paciente foi encaminhado para outro serviço odontológico, afim de realizar restaurações dentárias das unidades dentárias 16 e 46, e orientado a voltar anualmente ao Serviço de Cirurgia e Traumatologia para observar se houve recidiva do CO. Foi realizado um exame clinico minucioso afim de investigar possíveis alterações que caracterizassem a síndrome de Gorlin Goltz, entretanto, o paciente não apresentava lesões cutâneas compatíveis com carcinoma basocelular, hipertelorismo ocular e múltiplos CO, porém, como uma correta conduta clinica, o paciente foi orientado a procurar um médico para investigar possíveis associações relacionadas com alterações do gene PTCH, que característico da síndrome de Gorlin Goltz. 


\section{Discussão}

O CO tem se apresentado como uma das entidades patológicas mais controversas da região oral e maxilofacial. Em comparação com outras lesões odontogênicas, os COs se destacam pelo comportamento clínico potencialmente agressivo, com tendência a recorrência local. A presença de múltiplos COs na região craniofacial e a alta taxa de recorrência estão associadas na maioria das vezes a Síndrome de Gorlin-Goltz, entretanto, no presente caso a lesão não estava associada a síndrome, visto que o paciente não apresentava múltiplos CO intraósseos, carcinoma basocelular, hipertelorismo ocular e costelas bífidas ${ }^{9}$.

A faixa etária de ocorrência do CO varia entre a $2^{a}$ e $3^{a}$ décadas de vida ${ }^{3}$. No presente estudo, a idade do paciente era 40 anos, sendo similar a outros estudos descritos na literatura $^{9,10}$. O CO é uma das lesões odontogênicas mais comuns que acometem a mandíbula, geralmente envolvendo ângulo e ramo ${ }^{10}$. No presente estudo, foi observado que a lesão estava presente na região posterior da mandíbula, corroborando outros estudos.

O desenvolvimento do CO ocorre no sentido anteroposterior, sem causar expansão óssea evidente, tornando sua descoberta na maioria das vezes um achado radiográfico ${ }^{4,5}$. No exame de imagem do presente caso, observou-se uma lesão radiolúcida unilocular de contornos bem definidos, que inicialmente tinha a hipótese de cisto odontogênico de desenvolvimento, devido ao fato de ser uma lesão associada as raízes dentarias e os dentes acometidos apresentarem vitalidade pulpar, excluindo a hipótese de cistos de natureza inflamatória.

Recentes estudos multicêntricos, evidenciaram que 25 a 40 \% dos casos de CO estão associados à coroa de um dente incluso, logo, a falha da erupção dentária é clinicamente um fator constante ${ }^{1,3,4}$, no presente caso a lesão estava associada a raízes de elementos dentários erupcionados e com vitalidade pulpar positiva no teste de sensibilidade térmica.

Em relação ao tratamento, os COs podem ser tratados conservadoramente, por marsupialização e enucleação, tratamento mais conservador, ou enucleação seguida de ostectomia e uso de solução esclerosante de Carnoy's, tratamento mais agressivo, em lesões recorrentes ou com maiores diâmetros ${ }^{8}$. No presente caso, foi realizada a exodontia do elemento dentário envolvido, as unidades 37 e 38 e inserido um dispositivo intraósseo para que ocorresse a descompressão da lesão cística. A literatura aponta que a marsupialização diminui a probabilidade de recorrência, sendo vantajosa especialmente em casos de lesões extensas, que durante a descompressão, ocorre um espessamento da cápsula fibrosa, facilitando a completa remoção cirúrgica da lesão, como observado no presente caso, que a lesão remanescente foi removida após seis meses, após ter regredido o seu tamanho 9 . O paciente foi colaborativo em relação a higiene do local, não apresentando infecção durante o período em ficou instalado o dispositivo intraósseo, enfantizando que o tratamento realizado por marsupialização com inserção de um dispositivo intraósseo é eficaz para o sucesso do tratamento de lesões císticas com potencial agressivo, o paciente também foi orientado a voltar anualmente ao Serviço de Cirurgia e Traumatologia para observar se houve recidiva do CO.

\section{ConClusÃo}

Os achados do presente caso ressaltam a importância do diagnóstico clínico e radiográfico, pelo fato de exibir um diagnóstico diferencial com cistos ou tumores odontogênicos, merecendo destaque: ameloblastoma, mixoma, granuloma central de células gigantes, cisto dentígero, tumor odontogênico adenomatoide, fibroma ameloblástico, cisto ósseo traumático, granuloma central de células gigantes, cisto periodontal lateral e cisto de Gorlin, salientando que a ausência de expansão óssea do CO auxilia o diagnóstico diferencial com cisto radicular e cisto dentígero, como foi observado no presente caso. A literatura aponta 
outras opções de tratamentos mais agressivos como: ressecção ou enucleação complementada com solução de Carnoy, com ou sem ostectomia periférica, enucleação com curetagem óssea periférica ou ostectomia como método de manejo preferencial exibem menores chances de recidivas. O tratamento do $\mathrm{CO}$ no presente estudo foi o tratamento conservador, utilizando um dispositivo intraósseo para descomprimir a lesão cística, observando a regressão total da lesão, sem presença de recidiva do CO até o presente momento. É importante que seja realizado o acompanhamento clínico-radiográfico prolongado do paciente após a remoção do CO, entretanto, julgamos de suma importância a realização de outros estudos utilizando o tratamento conservador e com maior tempo de acompanhamento para a observação de possíveis recidivas da lesão.

\section{REFERÊNCIAS BIBLIOGRÁFICAS}

1. Deepthi PV, Beena VT, Padmakumar SK, Rajeev R, Sivakumar R. A study of 1177 odontogenic lesions in a South Kerala population. J Oral Maxillofac Pathol, 2016; 20 (2):202-4.

2. Menon S. Keratocystic odontogenic tumours: etiology, pathogenesis and treatment revisited. J Oral Maxillofac Surg Med Pathol, 2015; 14(3):541-7.

3. Osterne RL, Brito RG, Alves AP, Cavalcante RB, Sousa FB. Odontogenic tumors: a 5-year retrospective study in a Brazilian population and analysis of 3406 cases reported in the literature. Oral Surg Oral Med Oral Pathol Oral Radiol Endod, 2011; 111(4):474-81.

4. Gaitán-Cepada LA, Quezada-Rivera D, Tenorio-Rocha F, Leyva-Huerta ER. Reclassification of odontogenic karatocyst as tumour. Impact on the odontogenic tumours prevalence. Oral Dis, 2010; 16(2):185-7.

5. Barnes L, Eveson JW, Reichart P, SIdransky D. World Health Organization Classification of Tumours. Pathology and genetics of head and neck tumours IARC Press: Lyon, 2005; (6):283-327.

6. El-Naggar AK, Chan JKC, Grandis JR, Takata T and Slootweg PJ. World Health Organization classification of tumours. Pathology and genetics of head and neck tumours. Lyon: IARC Press 9, 2017; (7):80-3.

7. Azevedo RS, Cabral MG, dos Santos TC, de Oliveira AV, de Almeida OP, Pires FR. Histopathological features of keratocystic odontogenic tumor: a descriptive study of 177 cases from a Brazilian population. Int ] Surg Pathol, 2012; 20:154-60.

8. Bello IO. Keratocystic odontogenic tumor: A biopsy service's experience with 104 solitary, multiple and recurrent lesions. Med Oral, 2016: 1-3.

9. Dall'Magro AK, Ottini R, Lauxen ], Santos R, Valcanaia TC, Ávila V]B, Dall'Magro E. Síndrome de gorlin-goltzrelato de casos. RFO UPF, 2014; 19(2): 239-44.

10. Araújo FS, Rezende MN, Pereira PSS. Queratocisto: tratamento conservador e apresentação de dois casos clínicos. Rev Bras Cir Traumatol-BucoMaxilo-Fac, 2007; 4(1):22-8. 